Proceedings

\title{
Measurement of $\Lambda_{c}^{+}$Baryon Production in $\mathrm{p}-\mathrm{Pb}$ Collisions with ALICE at the LHC ${ }^{\dagger}$
}

\author{
Christopher Hills (1) on behalf of the ALICE Collaboration \\ Physics Department, University of Liverpool, Liverpool L69 7ZE, UK; christopher.hills@cern.ch \\ + Presented at Hot Quarks 2018-Workshop for Young Scientists on the Physics of Ultrarelativistic \\ Nucleus-Nucleus Collisions, Texel, The Netherlands, 7-14 September 2018.
}

Published: 12 April 2019

Abstract: Charmed baryons and their corresponding baryon-to-meson ratios are important tools to understand hadronisation processes in the Quark-Gluon Plasma produced in heavy-ion collisions. Recent Run 2 measurements in $\mathrm{p}-\mathrm{Pb}$ collisions at $5.02 \mathrm{TeV}$, performed with the ALICE experiment at the LHC, are presented and compared to theoretical model predictions.

Keywords: Heavy Ion Experiments; Quark-gluon plasma; heavy flavour

\section{Introduction}

Heavy quarks (charm, c and beauty, b) act as unique probes of the hot Quark-Gluon Plasma (QGP) medium produced in heavy-ion collisions. Due to their large masses with respect to the QCD energy scale, they are produced early in the collision in hard partonic scatterings. This means that they experience the full evolution of the medium and can provide information on transport mechanisms inside the QGP. Measurements of the baryon-to-meson ratio e.g., $\left(\Lambda_{c} / D^{0}\right)$ give sensitivity to the dominance of different hadronisation processes for charmed quarks. In the light flavour sector, an enhancement in this baryon-to-meson ratio has been observed at mid $p_{\mathrm{T}}(5 \mathrm{TeV} / \mathrm{c})$ for $p / \pi$ at RHIC [1] as well as for $\Lambda / K_{S}^{0}$ [2]. Two models which are able to explain this enhancement include the thermal model, where the hadrons are assumed to be produced at the QGP hadronisation phase and are in thermal equilibrium with the medium [3]. The second is through coalescence, where comoving partons combine their transverse momentum to form a final hadron with higher transverse momentum. Further enhancement is expected if [ud] diquarks exist in QGP [4].

While $\mathrm{Pb}-\mathrm{Pb}$ collisions form the basis of the QGP study at the LHC, measurements in smaller systems are necessary to give a full understanding of these collisions. pp collisions provide a baseline where a medium is not expected to be formed and thus act as a reference for the heavier collision systems. The expected lack of modification by a medium also makes these collisions a good test of predictions obtained with $\mathrm{pQCD}$ calculations. $\mathrm{p}-\mathrm{Pb}$ collisions are important to disentangle those effects due to a hot medium and cold nuclear matter effects caused by the inclusion of a nucleus in the initial state.

In these proceedings, we report on measurements of the production cross section of the charmed baryon $\Lambda_{c}$ [udc] in p-Pb collisions at $5.02 \mathrm{TeV}$, performed with the ALICE experiment. The corresponding baryon-to-meson ratio measurements are also presented, together with comparisons to theoretical model predictions.

\section{Data Analyses}

A description of the ALICE detector can be found here [5]. For the data analysis, three detectors located in the central barrel were used. The Inner Tracking System (ITS) is used for vertexing and track reconstruction. The Time Projection Chamber (TPC), the main tracking detector, also provides 
$d E / d x$ information for particle identification (PID). The Time Of Flight (TOF) detector provides further complementary PID information.

The measurement of $\Lambda_{c}$ production was performed by reconstructing two hadronic decay modes: $\Lambda_{c}^{+} \rightarrow p K_{s}^{0}$ with branching ratio (BR) $(1.58 \pm 0.08) \%$ and $\Lambda_{c}^{+} \rightarrow p K^{-} \pi^{+}$with BR $(6.35 \pm 0.33) \%$. In previous measurements with ALICE [6], a semileptonic channel $\Lambda_{c}^{+} \rightarrow e^{+} v_{e} \Lambda$ was also investigated in pp collisions at $\sqrt{s_{\mathrm{NN}}}=7 \mathrm{TeV}$. The $\Lambda_{c}^{+} \rightarrow p K_{s}^{0}$ decay channel was reconstructed from combining a bachelor track with a $K_{s}^{0}$ candidate. Whereas the $\Lambda_{c}^{+} \rightarrow p K^{-} \pi^{+}$decays were built up from triplets of reconstructed tracks with appropriate charge sign combinations. To reduce large combinatorial background, PID information from the TPC and TOF detectors was used. For the $p K_{s}^{0}$ decay channel, this was done with $n \sigma$ cuts around the proton hypothesis for the bachelor track. For the $p K^{-} \pi^{+}$ channel, a Bayesian approach [7] was used to combine the signals from the TPC and TOF detectors and assign conditional probabilities associated to different particle species $(\mathrm{p}, \mathrm{K}, \pi)$ to each track. The particle species with the highest probability is consequently assigned to that track.

Two different cut optimisation methods were used for signal extraction. The first relied on standard rectangular cuts on kinematic and geometric variables and this approach was tuned on Monte Carlo simulations to optimise the statistical significance. This is referred to as the standard (STD) method. A second method was also applied for the $p K_{s}^{0}$ decay channel using a multivariate approach with Boosted Decision Trees (BDTs) [8] and is referred to as the MVA method.

The analyses presented in these proceedings were performed on a dataset consisting of 600 million minimum bias events taken by ALICE at the LHC in 2016 for $\mathrm{p}-\mathrm{Pb}$ collisions at $\sqrt{s_{\mathrm{NN}}}=5.02 \mathrm{TeV}$. For the cut optimisation (STD method) and efficiency corrections, a Monte Carlo simulation consisting of 85 million events was used. These were generated using HIJING [9] and enriched with PYTHIA [10] events containing $c \bar{c}$ or b $\bar{b}$ pairs.

\section{Results}

In order to measure the charmed baryon $\left(\Lambda_{c}^{+}\right)$production cross section, several corrections must be applied to the raw yield, the most notable being the efficiency and acceptance corrections obtained from Monte Carlo simulations and the fraction of prompt $\Lambda_{c}$ given by FONLL calculations [11].

Figure 1 shows the prompt $\Lambda_{c}^{+} p_{\mathrm{T}}$-differential production cross section measured in $\mathrm{p}-\mathrm{Pb}$ collisions at $5.02 \mathrm{TeV}$ for each decay channel and both data analysis methods, as well as the combined results. The experimental $\Lambda_{c}^{+} / D^{0}$ ratio is also presented as a function of $p_{\mathrm{T}}$. It can be seen that all decay channels and signal extraction strategies yield consistent results within uncertainties and display a decreasing trend at high $p_{\mathrm{T}}$ for the $\Lambda_{c}^{+} / D^{0}$ ratio.
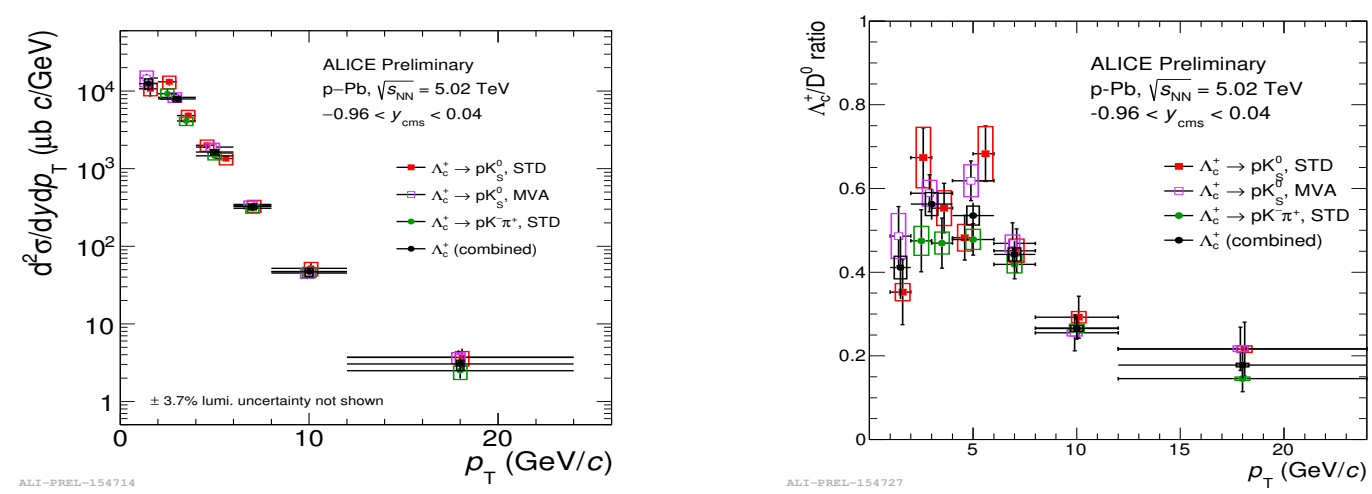

Figure 1. Prompt $\Lambda_{c}^{+}$baryon $p_{T}$ differential cross section (left) and $p_{\mathrm{T}}$-dependence of the $\Lambda_{c}^{+} / D^{0}$ ratio (right), measured in $\mathrm{p}-\mathrm{Pb}$ collisions at $5.02 \mathrm{TeV}$.

Figure 2 compares the results for the $\Lambda_{c}^{+}$cross section (combined for the two decay channels and analysis methods) and the baryon-to-meson ratio obtained from the data collected during the LHC 
Run 1 [6] and Run 2. The improved statistics of Run 2 allowed for the $p_{\mathrm{T}}$ range to be extended down to $1 \mathrm{GeV} / \mathrm{c}$ and up to $24 \mathrm{GeV} / \mathrm{c}$, as well as for a reduction of the statistical uncertainty.
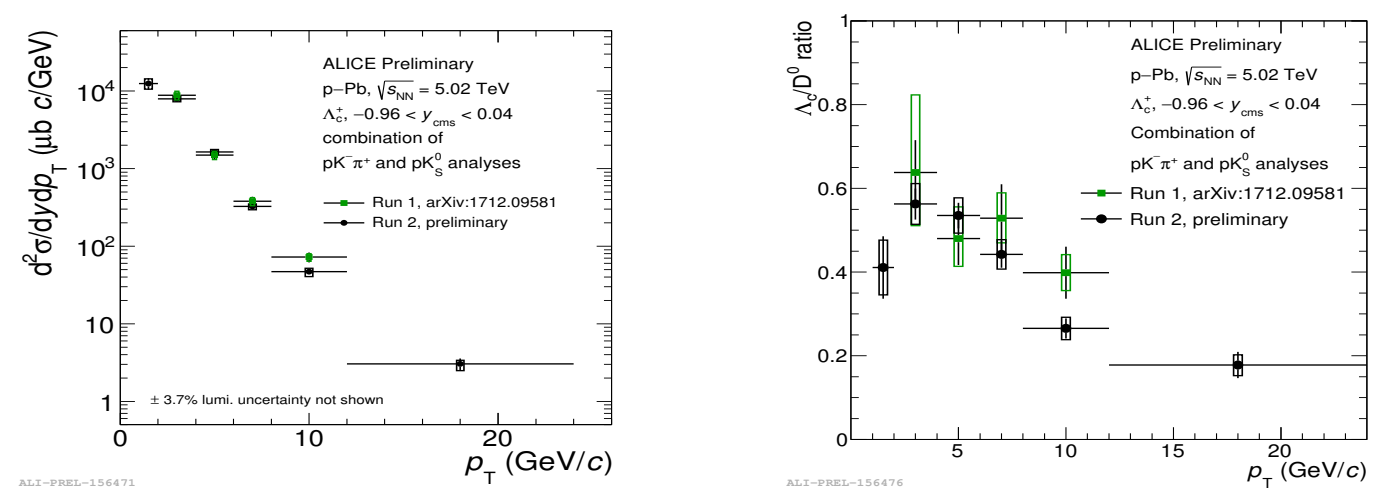

Figure 2. Comparison of the prompt $\Lambda_{c}^{+}$baryon $p_{T}$-differential cross section (left) and of the $p_{\mathrm{T}}$-dependence of $\Lambda_{c}^{+} / D^{0}$ ratio (right) measured in $\mathrm{p}-\mathrm{Pb}$ collisions at $5.02 \mathrm{TeV}$ for Runs 1 and 2 .

\section{Discussion}

The results shown in Figure 2 (left) are consistent with one another within uncertainties. This supports the earlier findings where the $\Lambda_{c}$ production was found to be underestimated by theoretical models [6].

In $\mathrm{p}-\mathrm{Pb}$ collisions, it is expected that there may be some modification due to the presence of the nucleus in the initial state. This is quantified by the nuclear modification factor, $\mathrm{R}_{p P b}$. This is calculated as the ratio of the production cross section in $\mathrm{p}-\mathrm{Pb}$ collisions with respect to $\mathrm{pp}$ collisions, scaled by the atomic mass number of the nucleus used. Plotted in Figure 3 are the nuclear modification factors measured for the $\Lambda_{c}$ baryon and D mesons at $5.02 \mathrm{TeV}$. The factors measured are found to be both consistent with each other and unity implying that there is little or no net modification due to cold matter effects. A comparison to theoretical models predictions is also presented, including POWHEG generated events + PYTHIA parton shower with EPS09 nuclear PDF [12] and a POWLANG transport model assuming a QGP is formed [13]. Current uncertainties on the measurements do not allow for any discrimination between the inclusion or exclusion of a hot QGP in $\mathrm{p}-\mathrm{Pb}$ collisions.

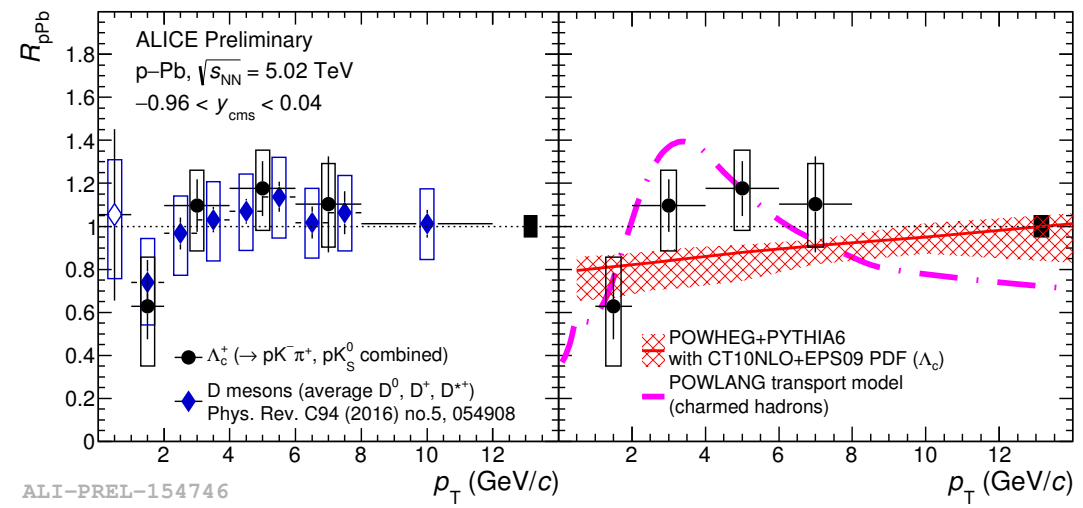

Figure 3. Nuclear modification factor $R_{\mathrm{pPb}}$ measured for $\Lambda_{c}$ and $\mathrm{D}$ mesons at $5.02 \mathrm{TeV}$ (left). Comparison to theoretical model predictions for $\Lambda_{c}$, see text (right).

The baryon-to-meson ratios measured for both heavy and (strange and non-strange) light-flavour particles are plotted in Figure 4 . The $p_{\mathrm{T}}$ distributions of these ratios appear to have a similar shape. The charm $\left(\Lambda_{c}^{+} / D^{0}\right)$ and strange $\left(\Lambda / p K_{s}^{0}\right)$ ratios seem to also be of similar magnitudes. This could suggest a common mechanism of hadronisation for strange and charm quarks in contrast to light, non-strange quarks in $\mathrm{pp}$ and $\mathrm{p}-\mathrm{Pb}$ collisions. 


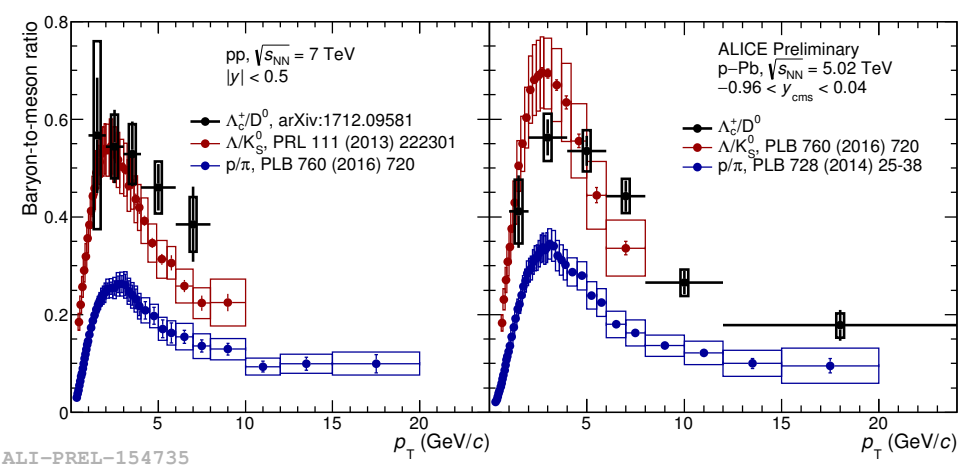

Figure 4. Comparison of the measured baryon-to-meson ratios $\Lambda_{c}^{+} / D^{0}, \Lambda / p K_{s}^{0}$ and $p / \pi$ in pp (left) and $\mathrm{p}-\mathrm{Pb}$ (right) collisions at $7 \mathrm{TeV}$ and $5.02 \mathrm{TeV}$, respectively.

\section{Conclusions}

The $\Lambda_{c}^{+}$production cross section and $\Lambda_{c}^{+} / D^{0}$ ratio were measured with ALICE using Run 2 data in $\mathrm{p}-\mathrm{Pb}$ collisions at $5.02 \mathrm{TeV}$. With improved statistics, a corresponding improvement to the $p_{\mathrm{T}}$ range and uncertainties was achieved over the Run 1 measurements. Current uncertainties still prevent any ability to distinguish between models with and without QGP formation in small systems. Although, there are some interesting qualitative similarities between heavy and light flavour baryon-to-meson ratios that will require further measurements to be fully understood.

Funding: This research was funded by Science and Technology Facilities Council grant number ST/N504142/1.

Conflicts of Interest: The author declares no conflict of interest.

\section{References}

1. Adcox, K.; Adler, S.S.; Ajitanand, N.N.; Akiba, Y.; Alexander, J.; Aphecetche, L.; Arai, Y.; Aronson, S.H.; Averbeck, R.; Awes, T.C.; et al. Suppression of Hadrons with Large Transverse Momentum in Central $A u+A u$ Collisions at $\sqrt{s_{\mathrm{NN}}}=130 \mathrm{GeV}$. Phys. Rev. Lett. 2001, 88, 022301-022307, doi:10.1103/PhysRevLett.88.022301.

2. Abelev, B.; Adam, J.; Adamova, D.; Adare, A.M.; Aggarwal, M.M.; Rinella, G.A.; Agnello, M.; Agocs, A.G.; Agostinelli, A.; Ahammed, Z.; et al. $K_{s}^{0}$ and $\Lambda$ Production in Pb-Pb Collisions at $\sqrt{s_{\mathrm{NN}}}=2.76$ TeV. Phys. Rev. Lett. 2013, 111, 222301-222311, doi:10.1103/PhysRevLett.111.222301.

3. Plumari, S.; Minissale, V.; Das, S.K.; Coci, G.; Greco, V. Charmed Hadrons from Coalescence plus Fragmentation in relativistic nucleus-nucleus collisions at RHIC and LHC. Eur. Phys. J. C 2018, 78, 348-361, doi:10.1140/epjc/s10052-018-5828-7.

4. Yasui, S.; Lee, S.H.; Ohnishi, K.; Yoo, I.K.; Koi, C.M. $\Lambda_{c}$ enhancement from strongly coupled QGP. Indian J. Phys. 2011, 85, 1043-1046, doi:10.1007/s12648-011-0142-3.

5. Aamodt, K.; Quintana, A.A.; Achenbach, R.; Acounis, S.; Adamová, D.; Adler, C.; Aggarwal, M.; Agnese, F.; Rinella, G.A.; Ahammed, Z.; et al. The ALICE experiment at the CERN LHC. J. Instrum. 2008, 3, S08002-S08261, doi:10.1088/1748-0221/3/08/S08002.

6. ALICE Collaboration. $\Lambda_{c}^{+}$production in pp collisions at $\sqrt{s_{\mathrm{NN}}}=7 \mathrm{TeV}$ and in $\mathrm{p}-\mathrm{Pb}$ collisions at $\sqrt{s_{\mathrm{NN}}}=5.02$ TeV. J. High Energy Phys. 2018, 2018, 108-147, doi:10.1007/JHEP04(2018)108.

7. Fragiacomo, E.; Šuljić, M.; Luparello, G.; Lea, R.; Camerini, P.; Margagliotti, G.; Piano, S.; Rui, R. Particle identification in ALICE: A Bayesian approach. Eur. Phys. J. Plus 2016, 131, 168-199, doi:10.1140/epjp/i2016-16168-5.

8. Voss, H. TMVA-The toolkit for multivariate data analysis. In Proceedings of the XIth International Workshop on Advanced Computing and Analysis Techniques in Physics Research (ACAT 2009), Saas-Fee, Switzerland, 11-15 March 2009; pp. 40-193.

9. Wang, X.N.; Gyulassy, M. HIJING: A Monte Carlo model for multiple jet production in pp, pA and A A collisions. Phys. Rev. D 1991, 44, 3501-3516, doi:10.1103/PhysRevD.44.3501. 
10. Sjostrand, T.; Mrenna, S.; Skands, P. PYTHIA 6.4 physics and manual. arXiv 2006, arXiv:hep-ph/0603175, doi:10.1088/1126-6708/2006/05/026.

11. Cacciari, M.; Frixione, S.; Houdeau, N.; Mangano, M.L.; Nason, P.; Ridolfi, G. Theoretical predictions for charm and bottom production at the LHC. J. High Energy Phys. 2012, 10, 137-159, doi:10.1007/JHEP10(2012)137.

12. Frixione, S.; Ridolfi, G.; Nason, P. A positive-weight next-to-leading-order Monte Carlo for heavy flavour hadroproduction. J. High Energy Phys. 2007, 9, 126-144, doi:10.1088/1126-6708/2007/09/126.

13. Beraudo, A.; De Pace, A.; Monteno, M.; Nardi, M.; Prino, F. Heavy-flavour production in high-energy d-Au and p-Pb collisions. J. High Energy Phys. 2016, 3, 123-145, doi:10.1007/JHEP03(2016)123.

(C) 2019 by the authors. Licensee MDPI, Basel, Switzerland. This article is an open access article distributed under the terms and conditions of the Creative Commons Attribution (CC BY) license (http:/ / creativecommons.org/licenses/by/4.0/). 